\title{
Properties and Semicrystalline Structure Evolution of Polypropylene/Montmorillonite Nanocomposites under Mechanical Load
}

Stribeck, Norbert; Zeinolebadi, Ahmad; Ganjaee Sari, Morteza; Botta, Stephan; Jankova Atanasova, Katja; Hvilsted, Søren; Drozdov, Aleksey; Klitkou, Rasmus; Potarniche, Catalina-Gabriela; Christiansen, Jesper deClaville

Total number of authors:

11

Published in:

Macromolecules

Link to article, DOI:

$10.1021 / \mathrm{ma202004f}$

Publication date:

2012

Document Version

Publisher's PDF, also known as Version of record

Link back to DTU Orbit

Citation (APA):

Stribeck, N., Zeinolebadi, A., Ganjaee Sari, M., Botta, S., Jankova Atanasova, K., Hvilsted, S., Drozdov, A.,

Klitkou, R., Potarniche, C-G., Christiansen, J. D., \& Ermini, V. (2012). Properties and Semicrystalline Structure Evolution of Polypropylene/Montmorillonite Nanocomposites under Mechanical Load. Macromolecules, 45(2), 962-973. https://doi.org/10.1021/ma202004f

\section{General rights}

Copyright and moral rights for the publications made accessible in the public portal are retained by the authors and/or other copyright owners and it is a condition of accessing publications that users recognise and abide by the legal requirements associated with these rights.

- Users may download and print one copy of any publication from the public portal for the purpose of private study or research.

- You may not further distribute the material or use it for any profit-making activity or commercial gain

- You may freely distribute the URL identifying the publication in the public portal 


\title{
Properties and Semicrystalline Structure Evolution of Polypropylene/ Montmorillonite Nanocomposites under Mechanical Load
}

\author{
Norbert Stribeck, ${ }^{* \dagger}$ Ahmad Zeinolebadi, ${ }^{\dagger}$ Morteza Ganjaee Sari, ${ }^{\ddagger}$ Stephan Botta, ${ }^{\S}$ Katja Jankova,"
}

Søren Hvilsted," Aleksey Drozdov, ${ }^{\perp}$ Rasmus Klitkou, ${ }^{\#}$ Catalina-Gabriela Potarniche, ${ }^{\#}$ Jesper deClaville Christiansen, ${ }^{\#}$ and Valentina Ermini ${ }^{\&}$

${ }^{\dagger}$ Institute TMC, Department of Chemistry, University of Hamburg, Bundesstr. 45, D-20146 Hamburg, Germany

${ }^{\ddagger}$ Department of Polymer Engineering and Color Technology, Amirkabir University of Technology, P.O. 15875-4413, 424 Hafez Ave., Tehran, Iran

${ }^{\S}$ HASYLAB at DESY, Notkestr. 85, D-22603 Hamburg, Germany

"Danish Polymer Center, Department of Chemical and Biochemical Engineering, Technical University of Denmark, Building 227, DK-2800 Kgs. Lyngby, Denmark

${ }^{\perp}$ Danish Technological Institute, Gregersensvej 1, DK-2630 Taastrup, Denmark

\#Department of Production, Aalborg University, Fibigerstræde 16, DK-9220 Aalborg, Denmark

${ }^{\&}$ Laviosa Chimica Mineraria S.P.A., Via Galvani 20, I-57123 Livorno, Italy

ABSTRACT: Small-angle X-ray scattering (SAXS) monitors tensile and load-cycling tests of metallocene isotactic polypropylene (PP), a blend of PP and montmorillonite (MMT), and two block copolymer compatibilized PP/MMT nanocomposites. Mechanical properties of the materials are similar, but the semicrystalline nanostructure of the PP differs. This is explained by a nucleation effect of the MMT. Competitive crystal growth diminishes crystallite sizes. The reinforcing effect of the MMT filler appears consumed by weakening the PP matrix. Decays

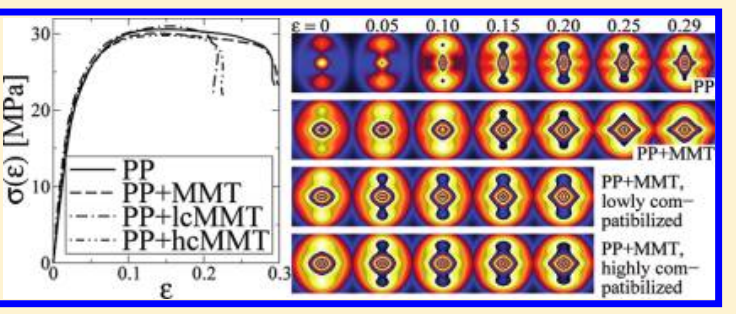
of mechanical and nanostructure response in dynamic load cycling indicate materials fatigue. Lifetimes describe the reinforcing and weakening effects. Addition of $3 \%$ MMT halves the fortifying effect of the PP nanostructure. A net gain of reinforcement (11\%) is observed with the highly compatibilized composite in which the strength of the semicrystalline PP is reduced to $25 \%$. Other results concern the evolution of Strobl's block structure and void formation during tensile loading.

\section{INTRODUCTION}

Polypropylene as Base Material of Composites. Polypropylene $^{1}(\mathrm{PP})$ is the material preferred ${ }^{2}$ by automotive industry for replacement of metal by plastics for reduction of weight and fuel consumption. As it comes to weight reduction of load-bearing components, the materials properties of the polymer are insufficient. A solution of this problem is the use of hybrid modules from PP and metal. Such modules are expensive. One could reduce the cost if one would succeed to develop an easily processable, low-fatigue PP-based composite. Glass-fiber reinforced PP composites ${ }^{3,4}$ are well introduced and exhibit advanced properties. Nevertheless, the production of the molded parts is still elaborate because at least the rupture of the glass fibers must be minimized. Thus, layered silicates ${ }^{5-12}$ bonded with PP could become an economical alternative, as is pointed out in recent reviews on polymer nanocomposites. ${ }^{13,14}$

Polypropylene as a Special Semicrystalline Material. Polypropylene $^{1,15}$ is one of the many thermoplastics that are semicrystalline at service temperature. Their properties depend on the semicrystalline morphology. Joined crystallites form crystalline lamellae. Crystalline and amorphous lamellae pile up, and these stacks are the building blocks of spherulites. This multiscale morphology is generated by crystallization from the melt. Whereas many polymers start to crystallize when the melt is moderately cooled below the melting temperature, pure PP only starts to crystallize when it is undercooled by more than $50{ }^{\circ} \mathrm{C} .{ }^{5,16}$ Consequently, PP is strongly susceptible to additives that change the crystallization behavior. Particularly with polypropylene the crystallization has frequently been found to propagate via a blocky mesophase. ${ }^{17,18}$ Moreover, in contrast to most polymers, polypropylene tends to form cross-hatched crystalline lamellae ${ }^{19-21}$ that connect the main lamellae and thus may build a 3-dimensional scaffold that reinforces the material. Ultimately, the many possible crystal modifications ${ }^{1}$ of PP can be used to optimize the grade with respect to application.

Nucleation of Technical Polypropylene. Delayed solidification is unacceptable for many manufacturing methods. Therefore, PP crystallization is accelerated by additives. Commercial

Received: September 2, 2011

Revised: November 6, 2011 
grades contain nucleators ${ }^{22-25}$ or clarifiers that nucleate the melt heterogeneously. During processing they support the creation of the required morphology. As such a grade and additional reinforcing components are bonded in a composite, ${ }^{26}$ the solidification process may be altered. ${ }^{5-10}$ Effects on the semicrystalline morphology cannot be excluded. Such effects may be investigated by small-angle X-ray scattering (SAXS). In particular, results from SAXS monitoring of structure evolution under load may advance the understanding of the relation between the composite's morphology and its practicality in a load-bearing application.

Monitoring Mechanical Tests. In service, materials are frequently subjected to strain or cyclic loading. Hence, resistance $^{27}$ to dynamic load (i.e., low fatigue ${ }^{28-30}$ ) is required. There is abundant experimental literature on the relation between materials structure and mechanical load. ${ }^{31}$ Nevertheless, the number of papers in which scattering is studied simultaneously during fatigue tests is still small. ${ }^{32,33}$ Fortunately, recent progress at synchrotron X-ray radiation facilities makes it possible to follow the variation of anisotropic scattering patterns of polymers during mechanical tests with sufficient accuracy.

\section{EXPERIMENTAL PART}

Materials. Nanocomposites from metallocene polypropylene (HM562S, LyondellBasell) and nanoclay (hydrophilic montmorillonite, MMT) are studied. The MMT is obtained from Laviosa Chimica Mineraria, Italy, as a $3.8 \mathrm{wt} \%$ aqueous dispersion. A compatibilizer is added to two of the materials in order to intercalate the MMT. The compatibilizer is an amphiphilic block copolymer $(\mathrm{AB})$ made by atom transfer radical polymerization (ATRP) ${ }^{34,35}$ It consists of a hydrophobic block of hydrogenated polybutadiene, i.e., poly(ethylene-co-1,2butylene) monoalcohol (PEB) (trade name: Kraton L-1203 from Kuraray Co., Japan) with molecular weight 7000 , PDI $=1.05$, and a hydrophilic block of quaternized dimethylaminoethyl methacrylate (DMAEMA) with 35 DMAEMA units. Modified nanoclays with 4.7 and $8.0 \mathrm{wt} \%$ of the $\mathrm{AB}\left(\mathrm{PEB}-b\right.$-PDMAEMA ${ }_{35} \mathrm{~N}^{+}$) have been prepared. For this reason $1660 \mathrm{~mL}$ (3.8 wt \%) of MMT dispersion was diluted with $1320 \mathrm{~mL}$ of distilled water under stirring at $80^{\circ} \mathrm{C}$ for $4 \mathrm{~h} .4 .5 \mathrm{~g}$ of $\mathrm{AB}$ in $450 \mathrm{~mL}$ of distilled water was added, and the mixture was stirred further at $40{ }^{\circ} \mathrm{C}$. The modified clay dispersion was filtered off and washed with water until no more $\mathrm{Na}^{+}$was detected $\left(0.1 \mathrm{M} \mathrm{AgNO}_{3}\right)$, mixed with water, and then freeze-dried. Table 1 describes the composition

Table 1. PP/MMT Nanocomposites Based on Metallocene Polypropylene (PP)-HM562S (LyondellBasell) and Montmorillonite (MMT) (Laviosa) ${ }^{a}$

\begin{tabular}{ll}
\multicolumn{1}{c}{ sample } & \multicolumn{1}{c}{ composition } \\
$\mathrm{PP}$ & pure polypropylene \\
$\mathrm{PP}+\mathrm{MMT}$ & $\mathrm{PP}+3 \mathrm{wt} \%$ freeze-dried MMT \\
$\mathrm{PP}+\mathrm{lcMMT}$ & $\mathrm{PP}+(3 \mathrm{wt} \% \mathrm{MMT}+4.7 \mathrm{wt} \% \mathrm{AB})$ \\
$\mathrm{PP}+$ hcMMT & $\mathrm{PP}+(3 \mathrm{wt} \% \mathrm{MMT}+8.0 \mathrm{wt} \% \mathrm{AB})$
\end{tabular}

${ }^{a}$ The compatibilizer is an amphiphilic block copolymer $(\mathrm{AB})$.

of the studied materials. Sample PP is the pure polypropylene. Sample $\mathrm{PP}+\mathrm{MMT}$ is a blend of polypropylene and the freeze-dried hydrophilic MMT. The samples PP + lcMMT and PP + hcMMT are composites that contain MMT with low compatibilizer amounts and high compatibilizer amounts, respectively. In order to prepare the nanocomposites, modified lcMMT and hcMMT have first been freezedried and, second, blended with the PP.

Test bars S2 according to DIN 53504 are injection molded in a MiniJet II (Thermo Scientific) from a melt of $200{ }^{\circ} \mathrm{C}$. Mold temperature: $30{ }^{\circ} \mathrm{C}$, molding pressure: $650 \mathrm{bar}$, molding time: $45 \mathrm{~s}$; holding pressure: $100 \mathrm{bar}$; holding time: $20 \mathrm{~s}$. The cross section of the parallel central part is $4 \mathrm{~mm} \times 2 \mathrm{~mm}$.
Tensile Testing. Tensile testing is performed in a self-made ${ }^{36}$ machine. A grid of fiducial marks is printed on the test bars. ${ }^{37}$ The clamping distance is $45 \mathrm{~mm}$. A $500 \mathrm{~N}$ load cell is used. Signals from load cell and transducer are recorded during the experiment. The sample is monitored by a TV camera. Video frames are grabbed every $10 \mathrm{~s}$ and stored together with the experimental data. The machine is operated at a cross-head speed of $0.5 \mathrm{~mm} / \mathrm{min}$. Using the fiducial marks, the local strain $\varepsilon=\left(l-l_{0}\right) / l_{0}$ is computed automatically ${ }^{38}$ from the average initial distance, $l_{0}$, of the fiducial marks and the respective actual distance, $l$. The true stress, $\sigma=F / A$, is computed from the force $F$ measured by the load cell after subtracting the force exerted by the upper sample clamp, and $A=A_{0} /(1+\varepsilon)$, the estimated actual sample cross section. $A_{0}$ is the initial cross section of the central zone of the test bar. The equation assumes conservation of sample volume.

In the continuous straining experiments necking starts after a draw path of ca. $3.5 \mathrm{~mm}$. The experiment is stopped after the neck is fully developed. In the load-cycling experiments the samples are prestrained by $2 \mathrm{~mm}$. After that the cycling starts. In each cycle the samples are strained by $1 \mathrm{~mm}$ and thereafter retracted by the same draw path.

SAXS Setup. Small-angle X-ray scattering (SAXS) is carried out in the synchrotron beamline A2 at HASYLAB, Hamburg, Germany. The wavelength of radiation is $\lambda=0.15 \mathrm{~nm}$, and the sample-detector distance is $3031 \mathrm{~mm}$. Scattering patterns are collected by a 2D marccd 165 detector (mar research, Norderstedt, Germany) in binned $1024 \times$ 1024 pixel mode (pixel size: $158.2 \mathrm{~m} \times 158.2 \mathrm{~m}$ ). Scattering patterns are recorded every $30 \mathrm{~s}$ with an exposure of $20 \mathrm{~s}$. The scattering patterns are normalized and background corrected. ${ }^{39}$ This means intensity normalization for constant primary beam flux, zero absorption, and constant irradiated volume $V_{0}$. Because the flat samples are wider than the primary beam, the correction has been carried out assuming $V(t) / V_{0}=(1 /(1+\varepsilon(t)))^{0.5}$. The equation assumes constant sample volume.

SAXS Data Evaluation. The scattering patterns $I(\mathbf{s})=I\left(s_{12}, s_{3}\right)$ are transformed into a representation of the nanostructure in real space. The only assumption is presence of a multiphase topology. The result is a multidimensional chord distribution function (CDF), $z(\mathbf{r}){ }^{40}$ The method is exemplified in a textbook (ref 39, Sect. 8.5.5) and in the original paper ${ }^{40}$ where figures show the change of the pattern from step to step. Here we do not dwell on the exposition of the evaluation method but summarize the steps and introduce the important quantities. The CDF with fiber symmetry in real space, $z\left(r_{12}, r_{3}\right)$, is computed from the fiber-symmetrical SAXS pattern, $I\left(s_{12}, s_{3}\right)$, of a multiphase material. $\mathbf{s}=\left(s_{12}, s_{3}\right)$ is the scattering vector with its modulus defined by $|\mathbf{s}|=s=(2 / \lambda) \sin \theta .2 \theta$ is the scattering angle. In order to compute $z\left(r_{12}, r_{3}\right), I\left(s_{12}, s_{3}\right)$ is projected on the representative fiber plane. Multiplication by $s^{2}$ applies the real-space Laplacian. The density fluctuation background is determined by low-pass filtering. It is eliminated by subtraction. The resulting interference function, $G\left(s_{12}, s_{3}\right)$, describes the ideal multiphase system. Its $2 \mathrm{D}$ Fourier transform is the sought CDF. In the historical context the CDF is an extension of Ruland's interface distribution function (IDF) ${ }^{41}$ to the multidimensional case or, in a different view, the Laplacian of Vonk's multidimensional correlation function. ${ }^{42}$ The CDF is an "edgeenhanced autocorrelation function" ${ }^{\text {43-46 }}$ - the autocorrelation of the gradient field, $\nabla \rho(\mathbf{r}) . \rho(\mathbf{r})$ is the electron density inside the sample that is constant within a domain (crystalline, amorphous). Thus, as a function of ghost displacement $\mathbf{r}$, the multidimensional $\operatorname{CDF} z(\mathbf{r})$ shows peaks wherever there are domain surface contacts between domains in $\rho\left(\mathbf{r}^{\prime}\right)$ and in its displaced ghost $\rho\left(\mathbf{r}^{\prime}-\mathbf{r}\right)$. Such peaks $h_{i}\left(r_{12}, r_{3}\right)$ are called ${ }^{41}$ distance distributions. Distance $\mathbf{r}=\left(r_{12}, r_{3}\right)$ is the ghost displacement. Sometimes it is useful to replace the index $i$ in $h_{i}\left(r_{12}, r_{3}\right)$ by a sequence of indices that indicate the sequence of domains that have been passed along the displacement path until the considered domain surface contact occurs. For example, $h_{c a}\left(r_{12} r_{3}\right)$ indicates the passing of an amorphous and a crystalline domain. Thus, this peak is a long-period peak. $h_{c a}$ will be used if the displacement is in the direction of strain. $h_{b a}$ will be used if the displacement is in the lateral direction (i.e., transverse to strain), where $b$ indicates the width of a block according to Strobl's block structure. ${ }^{17,18}$ 
From the oriented scattering pattern we compute the scattering power $Q$ of the ideal semicrystalline morphology (no density fluctuations within the domains, no density transition zone between the domains) ${ }^{39}$ For this purpose we start from $G\left(s_{12}, s_{3}\right)$ and extract the scattering intensity $I_{i d}(\mathbf{s})$ of the ideal semicrystalline morphology. Then $\left\{I_{i d}\right\}\left(s_{1}, s_{3}\right)=\int I_{i d}\left(s_{1}, s_{2}, s_{3}\right) \mathrm{d} s_{3}$ is computed. The equation presents the definition of the projection operation. $\left\{I_{i d}\right\}\left(s_{1}, s_{3}\right)$ is the scattering intensity of the ideal multiphase system projected on the representative $s_{13}$-plane of fiber symmetry. ${ }^{39}$ From $\left\{I_{i d}\right\}\left(s_{1}, s_{3}\right)$ the scattering power

$$
Q=\iint\left\{I_{i d}\right\}\left(s_{1}, s_{3}\right) \mathrm{d} s_{1} \mathrm{~d} s_{3}
$$

is directly computed. $Q$ is normalized with respect to the irradiated volume because of the respective normalization of $I(\mathbf{s})$. In order to relate changing $Q$ to structure evolution, it is helpful to know where the scattering intensity is changing. In general, the presentation of variations in images $\left\{I_{i d}\right\}\left(s_{1}, s_{3}\right)$ is difficult, but if the intention is a discrimination between, e.g., void formation and change of the semicrystalline structure, an isotropic scattering curve $I(s)$ can be utilized. From $\left\{I_{i d}\right\}\left(s_{1}, s_{3}\right)=\left\{I_{i d}\right\}(s, \phi)$ an isotropic scattering curve

$$
4 \pi s^{2} I(s)=\int_{0}^{2 \pi}\left\{I_{i d}\right\}(s, \varphi) \mathrm{d} \varphi
$$

is obtained by circular integration of $\left\{I_{i d}\right\}(s, \phi) \mathrm{d} \phi$ with respect to the polar angle $\phi$ in the $s_{13}$-plane. Of course, further integration with respect to $s$ yields the scattering power. Thus, it appears reasonable to inspect the integrand $s^{2} I(s)$ in order to determine which angular regime is responsible for observed changes in $Q$.

\section{RESULTS}

3.1. Tensile Tests. A self-made ${ }^{36}$ tensile tester that performs symmetric drawing is used. Thus, the same spot of the sample is monitored by the X-ray, as long as the sample is homogeneously extended. If the tested material starts to neck, a peculiar problem is encountered that limits the interval in which data evaluation appears reasonable. This is demonstrated in Figure 1. In the inset images the irradiated spot is indicated by a cross. As the material begins to neck (Figure 1, inset c),

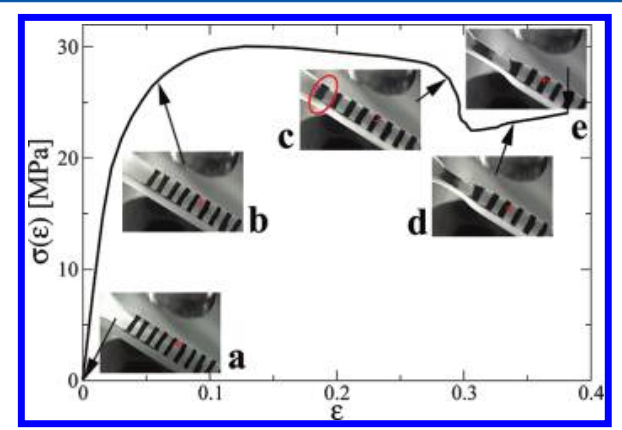

Figure 1. Stress relaxation and spot translation caused by necking. True stress $\sigma(\varepsilon)$ as a function of local strain $\varepsilon$. (a) Start of test. The Xray beam spot is indicated by a cross. (b) Homogeneous stretching. Spot does not move. (c) Necking has started (see ellipse). Material shows stress relaxation. (d) Spot moving toward neck. (e) End of experiment.

stress relaxation is observed and the irradiated spot starts to move along the sample toward its neck. Because the X-ray is no longer monitoring the same location of the material, the corresponding data are not discussed.

Figure 2 shows the mechanical data of the tensile tests in the synchrotron beam. The true stress $\sigma$ is plotted as a function of the local strain $\varepsilon$ measured at the point of irradiation by the

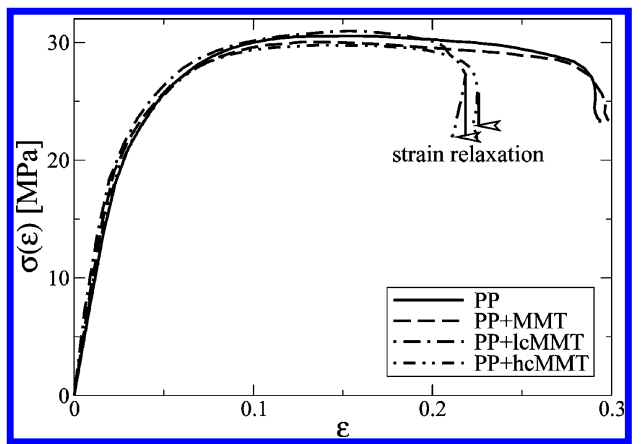

Figure 2. Tensile testing of $\mathrm{PP} / \mathrm{MMT}$ materials in the synchrotron beam. True stress $\sigma(\varepsilon)$ as a function of the local strain $\varepsilon$ at the position of irradiation. Compatibilized nanocomposites exhibit even relaxation of local strain $\varepsilon$.

synchrotron beam. The spot-translation tail of the curves has been discarded. Obviously, the uncompatibilized materials (pure $\mathrm{PP}$ and $\mathrm{PP}+\mathrm{MMT}$ ) start necking at a higher elongation $(\varepsilon \approx 0.29)$ than the composites that contain compatibilized MMT. Moreover, these composites even show relaxation of the local strain while the neck is developing.

3.2. Discussion of Measured SAXS Patterns. Figure 3 presents central sections of selected SAXS patterns $I\left(s_{12}, s_{3}\right)$

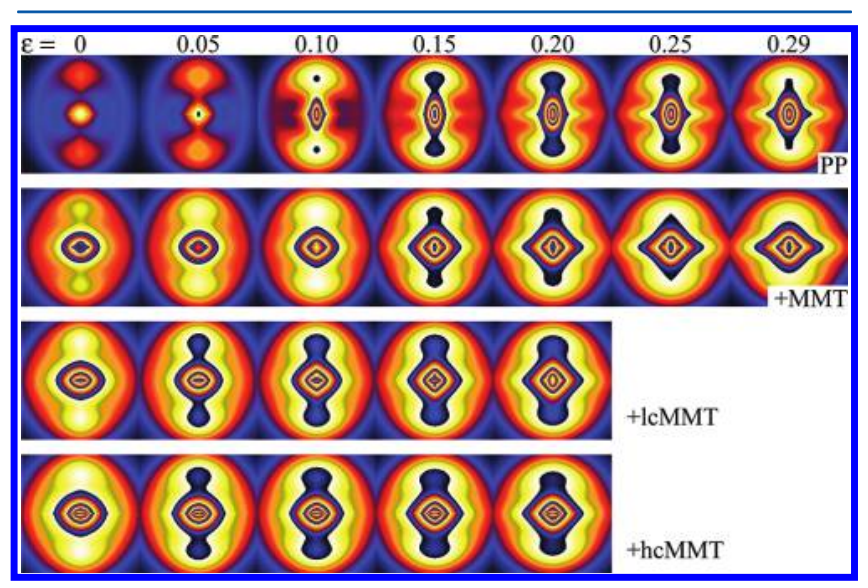

Figure 3. SAXS intensities $I\left(s_{12}, s_{3}\right)$ of PP, a blend (+MMT), and two composites (+lcMMT, +hcMMT) as a function of the local strain $\varepsilon$. Straining direction $s_{3}$ is vertical. The images are on the same logarithmic scale. They show the central region $-0.15 \mathrm{~nm}^{-1} \leq s_{12}, s_{3} \leq$ $0.15 \mathrm{~nm}^{-1}$ of the measured patterns in a repetitive pseudo-color presentation.

from the plain straining experiments as a function of the local strain $\varepsilon$. The measured images are larger and range to $s=0.25 \mathrm{~nm}^{-1}$. The logarithmic intensity scale is identical for all images.

Unstrained Materials. Before the start of the tests $(\varepsilon=0)$ all the injection-molded materials exhibit discrete SAXS with high uniaxial orientation. Two-point patterns are observed. The peak maxima are on the vertical axis $\left(s_{3}\right.$, meridian, direction of the melt flow in the bar, straining direction). From top to bottom both the peak intensity and the lateral peak width are strongly increasing. The narrow reflections of the pure PP (top) and of the blend PP + MMT (below) are indicative for layer stacks made from crystalline lamellae in the PP that extend in the direction perpendicular to the direction of melt flow. Nevertheless, the blend already contains an additional background. It is a broad peak underneath the narrow reflection, 
which indicates an additional microfibrillar component. In this component the shape of the crystalline domains is no longer an extended lamella, but only a granule of lower lateral extension. The blend and the composites exhibit strong central scattering of big scattering entities. These entities may either be crazes formed between the nanoparticles and the PP matrix or bigger particles of MMT that have not been exfoliated. Typical for craze scattering is diffuse central scattering showing an envelope of cross shape or of diamond shape. ${ }^{32,47-51}$

Diffuse Central Scattering as a Function of Strain. At $\varepsilon=0$ none of the samples exhibits diamond-shaped central scattering. From left to right in Figure 3 the local strain is increasing in steps of $\Delta \varepsilon=0.05$. All samples that contain MMT develop diamond-shaped central scattering during the test. This observation can be explained by voids in the MMT materials at least for $\varepsilon>0.15$. During the test even pure PP develops central scattering that is increasing up to $\varepsilon \approx 0.2$. Its envelope shows a different shape. It is an ellipse with its long axis in the direction of strain. This means that the corresponding scattering entities are oriented perpendicular to the straining direction. They could be explained by amorphous layers that are converted into crazes. In parallel, a rather well-defined equatorial streak is developing. Such streaks are typical for needle-shaped voids extended in the straining direction. ${ }^{52}$ In summary, the SAXS of all samples indicates void formation during tensile testing.

Pure Polypropylene. The top row of Figure 3 displays selected patterns taken during the tensile test of the pure PP material. The scale is identical for all pseudo-color images in the figure. With increasing local strain also the maximum peak intensity is increasing, and after the yield stress is reached (cf. Figure 2), i.e., $\varepsilon>0.1$, the peak itself is broadening in lateral direction. The peak broadening after reaching the yield-stress level indicates a decrease of crystallite extension in the direction perpendicular to the "fiber" axis. This decrease can be explained $^{53}$ by disruption of crystalline lamellae. Such a morphological transition from a lamellar to a microfibrillar stack is generally observed whenever semicrystalline polymers are subjected to uniaxial strain. ${ }^{54}$

PP/MMT Blend and Nanocomposites. The scattering patterns of the blend and the two nanocomposites are presented in rows 2-4 of Figure 3. All materials show the mentioned transition into a microfibrillar stack morphology, but their initial structure is different from the initial structure of the pure PP. The scattering patterns appear rather blurred, and a direct qualitative interpretation can only describe very general features. Thus, the scattering entities of the samples that contain MMT must be smaller, less homogeneous, and their arrangement less perfect than with the pure PP. A quantitative analysis will be based on the data after their transformation into the CDF that displays the morphological features more clearly.

3.3. Discussion of the CDF Patterns. Figure 4 shows the absolute values $\left|z\left(r_{12}, r_{3}\right)\right|$ of the CDFs for the four studied materials. The pseudo-color scale is identical for all images.

Nanostructure of the Unstrained Samples. The left column displays the CDFs of the samples at $\varepsilon=0$. Most prominent are the layerlike peaks on the meridian of the CDFs. They describe the stacked crystalline and amorphous layers of the polypropylene matrix and their correlation in straining direction. Their lateral extension is a measure of the average lateral extension of the layers. These reflections are analyzed quantitatively in section 3.5 .

The CDF in the top left corner shows the pure PP. Compared to the blend and the composites below, its peaks are wider in

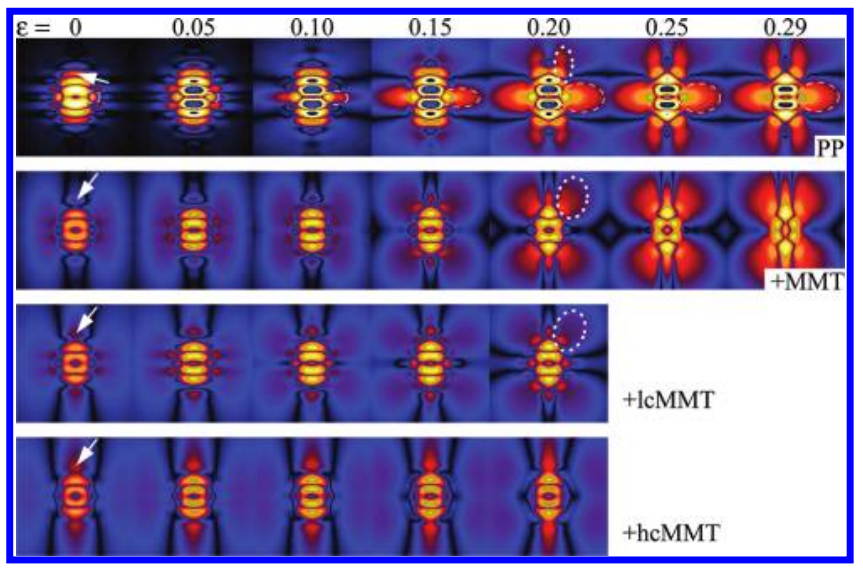

Figure 4. Absolute values $\left|z\left(r_{12}, r_{3}\right)\right|$ of chord distribution functions (CDF) of PP, a blend (+MMT), and two composites (+lcMMT, +hcMMT) as a function of the local strain $\varepsilon$. Straining direction $r_{3}$ is vertical. The images are on the same logarithmic scale. In a repetitive pseudo-color representation the images show the region $-60 \mathrm{~nm} \leq$ $r_{12}, r_{3} \leq 60 \mathrm{~nm}$ of the patterns computed from SAXS data by a special Fourier transform.

horizontal direction. Thus, the lamellae are wider than those of the samples that contain MMT. Moreover, several distinct reflections are piled up. This shows that the thickness variation of the layers is low, and at least three crystalline lamellae are correlated in each stack. In the samples containing MMT the correlation along the stack is even lower. There are only two crystalline layers in each stack (a "sandwich"). Arrows point at the shape distributions $h_{c a c}\left(r_{12}, r_{3}\right)$ of such sandwiches. For the pure PP $h_{c a c}\left(r_{12}, r_{3}\right)$ is narrow in the $r_{3}$-direction. Thus, the heights of all sandwiches are almost the same. In the lateral $r_{12}{ }^{-}$ direction the peak is extended. Thus, the sandwich is made from two crystalline lamellae that are well-aligned on top of each other.

In the materials that contain MMT the lateral width of $h_{c a c}\left(r_{12}, r_{3}\right)$ is narrower than the lateral width of the inner peaks. Thus, there is some lateral disorder in the piling. Let us discuss the shape of the sandwich peak in Figure 4 from top to bottom. In the $r_{3}$-direction the height distribution of the sandwiches becomes broader and more and more asymmetric. For the material with the high amount of compatibilizer (PP + hcMMT) the tail of the sandwich distribution even outreaches the limit of the image at $r_{3}=60 \mathrm{~nm}$. From top to bottom a decrease of domain size, domain uniformity, and domain arrangement is observed. This fact is as well reflected in the decrease of the sharp offmeridional peaks. They characterize correlations among crystallites the connecting line of which is not in straining direction.

All the CDFs of the MMT samples show an off-meridional, vertically extended intensity region. This intensity characterizes the distance distribution between the left and the right edge of microfibrils that house the crystalline domains. For pure PP this peculiar arrangement of crystalline domains is not observed.

Nanostructure Evolution in the Tensile Tests. In Figure 4 proceeding from left to right, the evolution of the semicrystalline polypropylene structure as a function of $\varepsilon$ is documented. For the pure PP (top row) the structure evolution is very much different from that of the other materials. Most peculiar is the development of a very strong equatorial long-period distribution $h_{b a}\left(r_{12}, r_{3}\right)$. In Figure 4, it is marked by a dashed-line ellipse. Already at $\varepsilon=0$ a pointed precursor peak is found on the equator. As the yield-stress level is reached at $\varepsilon=0.1$, the peak 
starts to grow outward on the equator (i.e., in the $r_{12}$-direction) by combining with an outer satellite. At $\varepsilon=0.2$ the peak widens in the $r_{3}$-direction, and from $\varepsilon \approx 0.25$ the shape of the peak does not change anymore. $h_{b a}$ describes a lateral correlation between adjacent crystallites that are no extended lamellae. Taking into account the satellite peaks that are visible at low strain, ensembles of three crystalline grains arranged along the $r_{12}$-direction are present. The corresponding scattering entity in the equatorial plane is readily identified as Strobl's block structure. ${ }^{17,18}$ In the patterns of samples containing MMT the block structure is much less distinct. Nevertheless, when the yield point is approached at $\varepsilon=0.1$, all materials exhibit a block structure. At this strain the three top patterns even show many sharp block-correlation peaks surrounding the center of the pattern. This observation indicates that close to the yield point the blocks even arrange ${ }^{55}$ in the third dimension. A threedimensional macrolattice ${ }^{56}$ of only short-range correlation has been formed.

Figure 5 presents results of a quantitative analysis of Strobl's block structure as a function of the local strain $\varepsilon$. The long

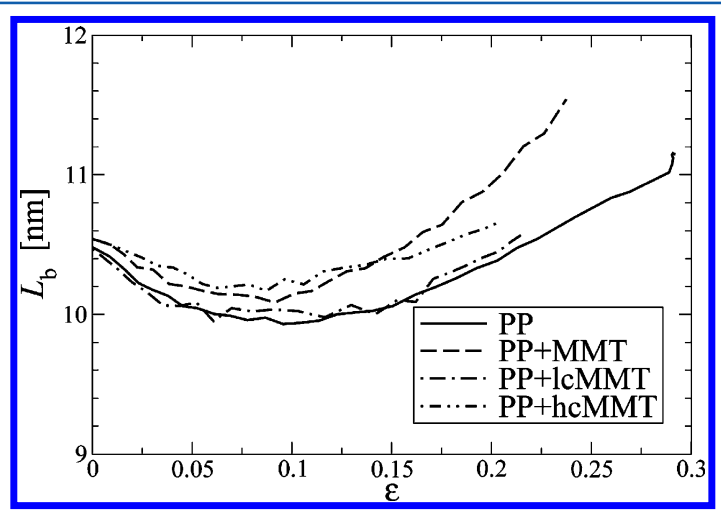

Figure 5. Evolution of the most-frequent long period $L_{b}$ of Strobl's block structure during tensile testing of PP and PP/MMT materials. $L_{b}$ is determined from the maximum position of the equatorial long period peak in the CDFs (cf. Figure 4).

period $L_{b}$ is shown. $L_{b}$ is the most-frequent distance between adjacent blocks in the equatorial $r_{12}$-plane as determined from the position of the maximum of $h_{b a}\left(r_{12}, r_{3}\right)$ in the CDF. For all samples $L_{b}$ decreases slightly with increasing $\varepsilon$ until yielding sets in. For $\varepsilon>0.1$ a slight increase is observed. Thus, the block structure itself behaves identical in all samples. The only difference is that addition of MMT diminishes its fraction severely.

Only for the pure PP with its strong block structure a more involved peak shape analysis is possible. As described in previous work, ${ }^{38,57}$ a bivariate polynomial is fitted to the cap of the peak in order to determine its position and the standard deviations that describe the peak widths in equatorial and meridional direction, respectively. Figure 6 presents the result. $L_{b}$ has already been discussed. $\Delta L_{b}$ is the breadth of $h_{b a}\left(r_{12}, r_{3}\right)$ in the equatorial $r_{12}$-direction. $H_{b}$ is the height of $h_{b a}\left(r_{12}, r_{3}\right)$ in the meridional $r_{3}$-direction. These two width parameters have been defined by 3 times the respective standard deviations. As has already been seen by inspection of the patterns, the breadth $\Delta L_{b}$ is increasing during the tensile test. The quantitative analysis shows that this increase is continuously slowed down with increasing $\varepsilon . H_{b}$ reflects the average height of the blocks. For low strain $H_{b}$ is constant at $5.5 \mathrm{~nm}$. Just before the yield level is reached at $\varepsilon \approx 0.08, H_{b}$ starts to increase linearly. The mechanism behind this growth of block height may be chain extension caused by the increased local stress around the blocks. Such chain extension could make the blocks grow tending toward a formation of extended chain crystals.

Some peaks in Figure 4 are difficult to analyze. They are encircled by dotted lines. These peaks result from sandwich domain size distributions $h_{c a c}\left(r_{12}, r_{3}\right)$ that overlap severely with the strong and narrow meridional distributions. Therefore, an analysis would require to separate these peaks from the meridional distributions by fitting the complete CDF patterns with a three-dimensional model of the semicrystalline PP nanostructure. The CDFs exhibit that such a model would have to be rather complex.

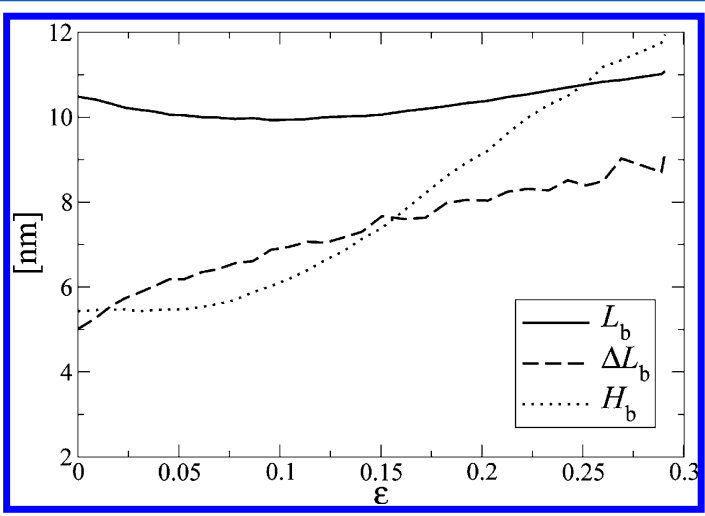

Figure 6. Nanostructure evolution of Strobl's block structure during tensile testing of the pure PP material from peak-shape analysis of the equatorial long-period peak $h_{b a}\left(r_{12}, r_{3}\right)$ in the CDF (encircled in Figure 4). $L_{b}$ is the most frequent distance between adjacent blocks, $\Delta L_{b}$ is the breadth of $h_{b a}\left(r_{12}, r_{3}\right)$, and $H_{b}$ is the height of $h_{b a}\left(r_{12}, r_{3}\right)$ in the straining direction.

3.4. Analysis of the Scattering Power. Figure 7 shows the evolution of the scattering power $Q$ (cf. eq 1) of the semicrystalline

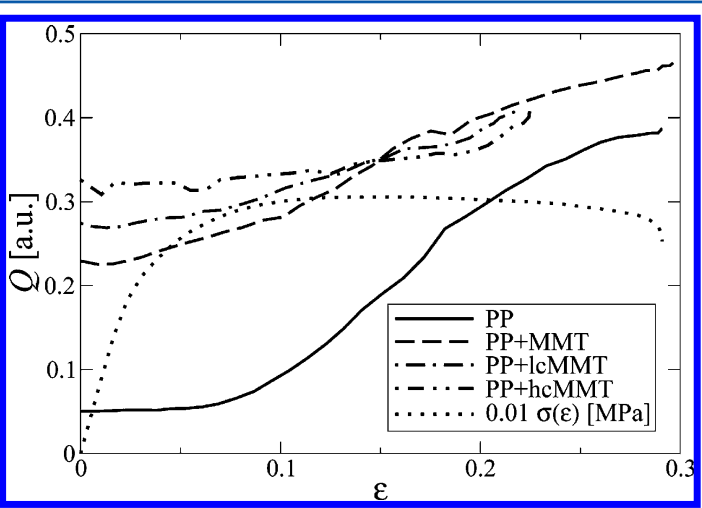

Figure 7. Evolution of the scattering power $Q$ during tensile testing of PP nanocomposites as a function of the local macroscopic strain $\varepsilon$ in the irradiated volume. Additionally, the stress-strain curve $\sigma(\varepsilon)$ of the pure PP material is shown.

PP as a function of strain. The curves are normalized to constant irradiated volume and constant flux. Thus

$$
Q c=\left(\rho_{c}-\rho_{a}\right)^{2} v(1-v)+X
$$

is valid with $c$ being a calibration constant. The contrast factor is specified by the electron densities $\rho_{c}$ and $\rho_{a}$ of the crystalline and the amorphous domains, respectively. $v$ is the volume crystallinity of the PP. Obviously, $v(1-v) \approx 0.24$ remains 

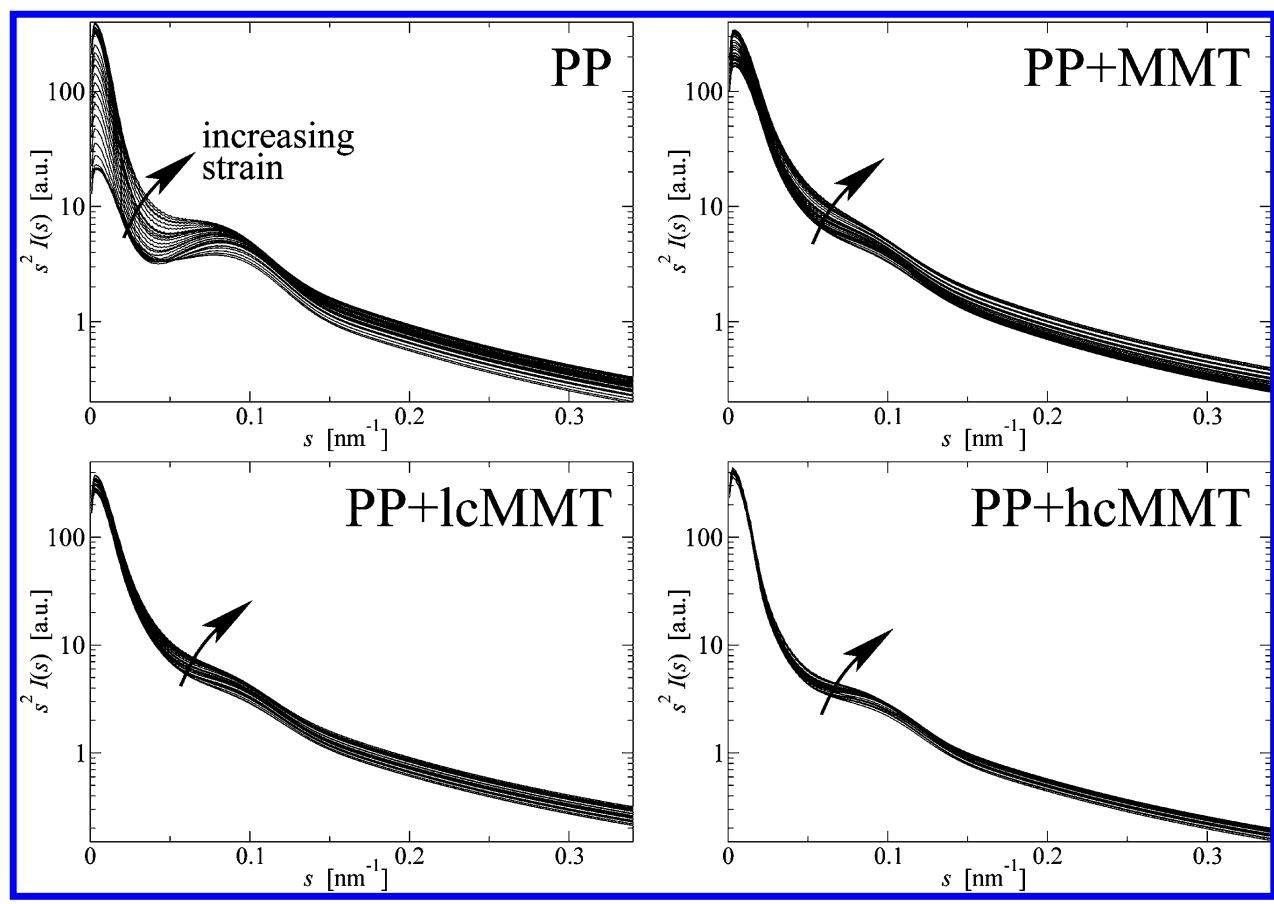

Figure 8. Evolution of the isotropic integrands $s^{2} I_{i d}(s)$ for the computation of $Q$ during tensile testing of PP nanocomposites as a function of the local macroscopic strain $\varepsilon$ on a logarithmic intensity scale.

almost constant for $0.3<v<0.7 . X$ is an unknown factor that describes both the scattering effects of MMT particles and of voids or crazes on $Q$. At $\varepsilon=0$ the scattering power of $P P$ is considerably smaller than that of the samples containing MMT. The reason is either the scattering tail of big MMT particles or the scattering of voids. Compatibilization of the MMT increases the scattering power even more. This result may be caused by MMT exfoliation in the nanocomposites.

For pure $\mathrm{PP} Q(\varepsilon)$ remains constant until the onset of yielding. The constant value for low strain is readily explained by constant contrast. After yielding sets in, the sample starts to become white. Thus, the strong increase of $Q(\varepsilon)$ for $\varepsilon>0.1$ can be related to void formation. It is well-known that voids mainly affect the scattering at low $s$. Because $Q$ is only a number, the isotropic scattering $s^{2} I(s)$ with $Q=4 \pi \int s^{2} I(s) \mathrm{d} s$ is prepared for angle-dependent inspection (Figure 8). For the sample PP Figure 8 shows a steep increase of scattering at very low angles $\left(s<0.05 \mathrm{~nm}^{-1}\right)$. On the other hand, the change of the long period peak $\left(s \approx 0.09 \mathrm{~nm}^{-1}\right)$ is only moderate. Thus, it can be concluded that the steep increase of $Q$ with sample PP indicates considerable formation of voids as the material is strained beyond the yield.

The blend PP + MMT exhibits high scattering at low $s$ even in the unstrained state. Further increase of low- $s$ scattering is moderate. This means that, in contrast to pure PP, straining of the blend does not induce an increase of voids that are small enough to be detected by SAXS.

For the nanocomposites PP + lcMMT and PP + hcMMT Figure 8 exhibits a similar response to strain, but the variation of the integrand with strain becomes even smaller. Thus, the effect of increasing compatibilization is an increased stability of the nanostructure of the polypropylene that has been formed during injection molding. Admittedly, this finding may simply mean that an already distorted structure cannot be destructed further. Another explanation would be based on a dynamic equilibrium that would not change the SAXS if the voids growing out of the SAXS detection window would be dynamically replaced by new small voids while all voids are continuously growing. In Figure 7, the moderate increase of the total $Q(\varepsilon)$ is depicted. The increase is fastest for the uncompatibilized blend and slowest for the composite that contains the high amount of compatibilizer. Whitening during straining has not been detected with the samples containing MMT, but detection is difficult anyway because the samples look brownish from the MMT.

3.5. Quantitative Analysis of Meridional Peaks. Figure 9 presents the evolution of the long period $L(\varepsilon)$. This is the most

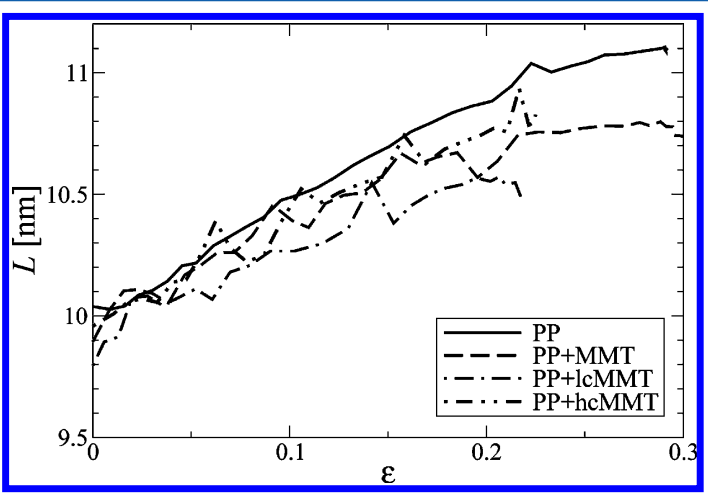

Figure 9. Nanostructure evolution during tensile testing of PP nanocomposites determined from the CDF long-period peak. Mostfrequently long period $L(\varepsilon)$.

frequent distance between two crystalline domains measured in straining direction.

At $\varepsilon=0$ the long periods of the four samples are identical. The increase of $L(\varepsilon)$ is much slower than expected from the macroscopic strain. At $30 \%$ strain $(\varepsilon=0.3) L$ has only grown by $10 \%$. The increase is somewhat slower for the samples containing MMT. The evolution of the peak shape (Figure 4) shows that an asymmetric long-period distribution is nonaffinely strained. 
Thus, the most probable $L$ is no measure of the average nanoscopic strain ${ }^{58}$ of the semicrystalline morphology (i.e., of the long period distribution $h_{c a}\left(r_{12}, r_{3}\right)$ that has its maximum at $\left.\left(r_{12}, r_{3}\right)=(0, L)\right)$.

The width $\Delta L$ of $h_{c a}\left(r_{12}, r_{3}\right)$ in the $r_{3}$-direction describes the heterogeneity of the stacking of crystalline and amorphous domains. Let $\Delta L=3 \sigma_{3}\left(h_{c a}\right)$ with $\sigma\left(h_{c a}\right)$ the standard deviation $h_{c a}$. Data are presented in Figure 10. Addition of MMT increases

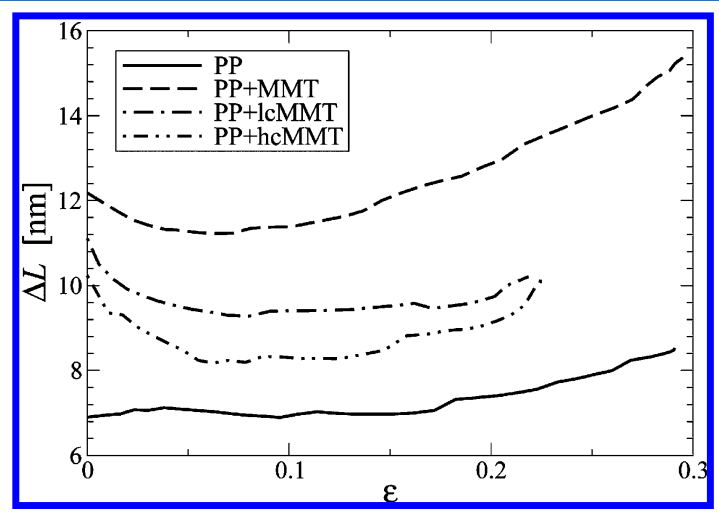

Figure 10. Nanostructure evolution during tensile testing of PP nanocomposites determined from the CDF long-period peak. Width $\Delta L(\varepsilon)$ in straining direction $r_{3}$ of the long period distribution $h_{c a}\left(r_{12}, r_{3}\right)$.

the heterogeneity of $h_{c a}$ considerably. Thus, the nanodomain stacking of PP is distorted by the MMT. Compatibilization leads to a relative reduction, indicating attenuation of the distorting effect of MMT on the semicrystalline structure.

With increasing strain the pure PP exhibits a moderate monotonous broadening. The MMT samples start with a slight homogenization of the stacks up to $\varepsilon \approx 0.07$ that is followed by a distinct loss of uniformity when the materials are above the yield. At high strain compatibilization $(\mathrm{PP}+\mathrm{lcMMT}, \mathrm{PP}+$ hcMMT) even further attenuates the distortion introduced by MMT. The asymmetry of $h_{c a}\left(r_{12}=0, r_{3}\right)$ is not considered and cannot be quantified from a peak fit that is based on a secondorder polynomial only.

The lateral extension $e_{12}(\varepsilon)=3 \sigma_{12}\left(h_{c a}\right)$ (in the $r_{12}$-direction) of $h_{c a}\left(r_{12}, r_{3}\right)$ measures the size of the crystallites in the transverse direction. $\sigma_{12}\left(h_{c a}\right)$ is the standard deviation of $h_{c a}$ in the $r_{12}$-direction. Figure 11 presents the results. $e_{12}(\varepsilon=0)$ is

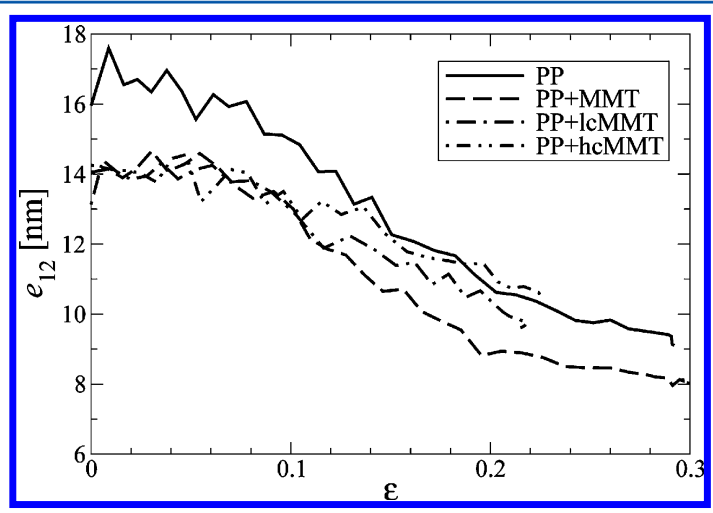

Figure 11. Nanostructure evolution during tensile testing of PP nanocomposites determined from the CDF long-period peak. Average lateral extension $e_{12}(\varepsilon)$ of the crystalline PP domains.

highest for the pure PP. Addition of MMT leads to a decrease of $e_{12}(0)$ by $2 \mathrm{~nm}$. The compatibilizer has no effect on the initial lateral extension of the crystalline domains, but on its decrease in the addressed conversion from layer stacks to microfibrillar $^{54,59,60}$ stacks. This decrease is generally observed ${ }^{55,61-66}$ during the straining of semicrystalline polymer materials. Compatibilization diminishes the degradation of the crystallite extension $e_{12}(\varepsilon)$ for $\varepsilon>0.1$, i.e., above the yield point.

3.6. Discussion of Nucleation and Compatibilization in the PP/MMT Nanocomposites. Nucleation. The depicted effects of clay on the semicrystalline morphology (broadening of the crystallite thickness distribution, smaller crystallite extension) may be explained by a strong nucleating effect of the MMT on polypropylene crystallization. Consequently, the nuclei density must have been increased, and during processing many crystallites grow simultaneously, mutually inhibiting their growth. Finally, the altered morphology is described by a less ordered arrangement of undersized, weak crystalline domains. The crystallinity is not increased. This means that addition of MMT to the commercial PP reduces the reinforcing effect of the crystalline polypropylene phase. In summary, part of the intrinsic reinforcement of MMT is not added to the strength of PP. Instead, it only replaces lost self-reinforcement of the polypropylene. As observed with the studied materials, the desired macroscopic reinforcement is not achieved. In section 3.7 we try to assess loss of self-reinforcement and gain of foreign reinforcement.

Nevertheless, although the mechanical properties of the four materials are very similar, monitoring of the straining experiments by SAXS has shown that the semicrystalline nanostructure and its evolution vary considerably. The relative variations from material to material indicate that the dominant troublesome effect of blending MMT, and the studied metallocene polypropylene grade is not the interfacial incompatibility between filler and matrix, but the alteration of the adjusted nanostructure of the matrix grade by the filler.

The addressed strong nucleating effect of MMT has been reported in several papers. ${ }^{5-9,11,67,68}$ According to Deshmane et al., ${ }^{10}$ the nucleating effect of MMT on polypropylene is high, whereas polyethylene remains almost unaffected. The addition of $4 \%$ MMT to polypropylene reduces the spherulite diameter from 210 to $14 \mu \mathrm{m}$, whereas the spherulite size of polyethylene remains unchanged. The different nucleating effect of MMT on polypropylene and polyethylene could explain why some of us have found ${ }^{69}$ a strong reinforcing effect of MMT on polyethylene.

On the other hand, Deshmane et al. ${ }^{10}$ report an increase of crystallite size with respect to the pure PP, whereas we and others ${ }^{9}$ find a decrease. According to the Deshmane paper, the addition of MMT is not accompanied by a decrease of mechanical properties. In contrary, the authors report an increase of Young's modulus by $50 \%$ and a considerable increase of the impact strength. An indication for the reason for the different results is the different crystallite size. The small and imperfect crystallites in our composites may be weaker than the extended crystallites grown in the composite prepared by Deshmane et al. ${ }^{10}$ A comparative study has reported ${ }^{5}$ that the alteration of polypropylene nanostructure by clay is not only a function of the pretreatment but also of the geological origin of the clay. Thus, the reason for the different findings may probably be the sensitive response of different polypropylene grades on different nucleating agents, MMT, and compatibilization. Consequently, our results indicate that blending a nucleating additive into a polypropylene grade may require reoptimization of its 
formulation. In a first step, one could try to reduce the supplierprovided nucleators $^{22-25}$ of the grade.

A decrease of crystallite size may be considered a decrease of filler particle size in the amorphous matrix. Theories of particulate reinforcement predict no influence of the filler size on the mechanical properties, although frequently an increase is empirically found. ${ }^{70}$ On the other hand, Sumita et al. ${ }^{71}$ report that for polypropylene also the opposite behavior can be observed. The reason may be that below a certain crystallite size further reduction will probably lead to weakening of the filler particle.

Compatibilization. The pathway to cure the shortcomings of a blend is compatibilization. The result is a nanocomposite. In our experiments the compatibilizer itself appears to inhibit crystal growth additionally (Figure 10 at $\varepsilon=0$ ). On the other hand, addition of more compatibilizer makes the nanostructure more stable when subjected to mechanical loading (Figures 3, 4, and 8). Admittedly, this stability is the stability of an already degraded structure. As shown by the necking-induced local strain relaxation (Figure 2), the compatibilizer increases the elasticity of the material.

3.7. Load-Cycling Experiments. Figure 12 shows the evolution of the true stress in the load-cycling experiments. The

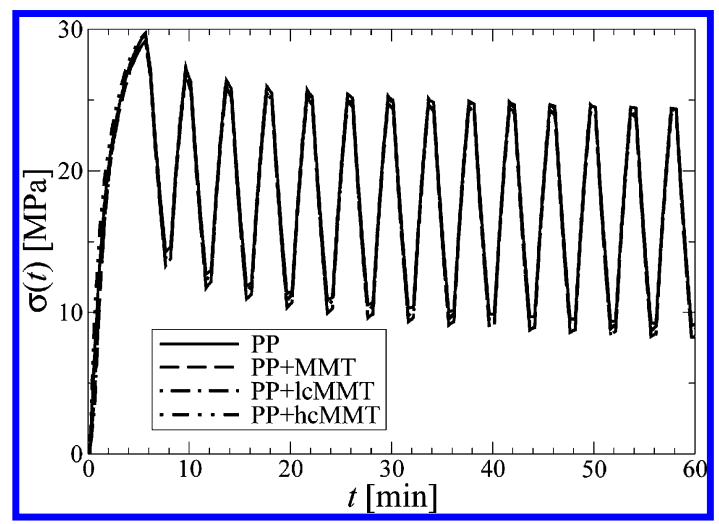

Figure 12. Slow load cycling of PP/MMT materials in the synchrotron beam. True stress $\sigma(t)$ as a function of the time $t$.

local strain $\varepsilon(t)$ is the dynamic stimulus. It is identical for all materials and presented in some of the following figures. Macroscopic fatigue of the materials is indicated by the decay of the peak stresses from cycle to cycle. Among the four materials the difference appears small. In order to quantify the macroscopic fatigue, the decay of the peak stresses has been evaluated quantitatively. Figure 13 shows the data in a semilogarithmic plot. In order to linearize the data, a residual stress $\sigma_{r}$ has been subtracted. From the add-on decay the lifetime of macroscopic stress fatigue, $\tau_{\sigma}$ has been computed by logarithmic regression (results in Table 2).

The nanoscale response of the material is evaluated from the long-period peak of the CDFs that have been computed from the SAXS patterns. The evolution of the most-probable long periods $L$ during load cycling is presented in Figure 14. The measured amplitudes are very low because the elongation of $h_{c a}\left(r_{12}, r_{3}\right)$ in the $r_{3}$-direction is nonaffine (see section 3.5). For all materials the responses $L(t)$ are in phase with the stimulus $\varepsilon(t)$. This has been expected because of the low load-cycling frequency. Nanostructure fatigue is manifested in the underlying decay of $L(t)$. The fit of the peak $L$ values with an exponential function (Figure 15) yields a quantitative assessment of

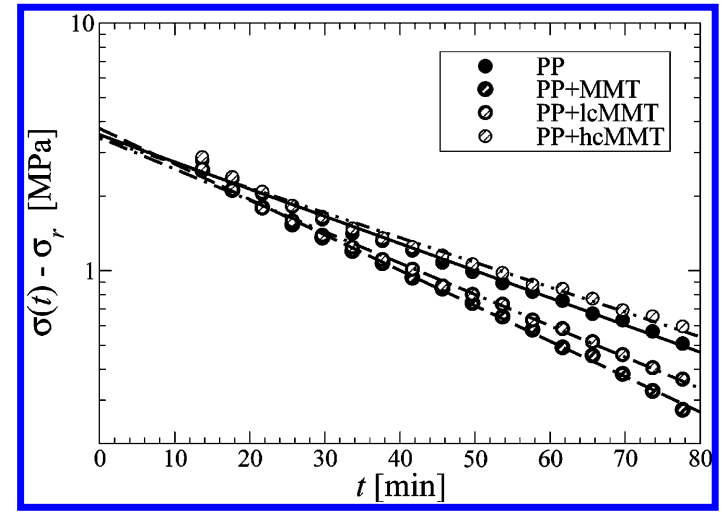

Figure 13. Slow load cycling of PP/MMT materials. Assessment of fatigue by exponential regression using the oscillation maxima of Figure 12. In order to linearize the plot $\sigma_{r}=23.6 \mathrm{MPa}$ was subtracted for PP and PP + hcMMT. $\sigma_{r}=23.4 \mathrm{MPa}$ linearizes the data of $\mathrm{PP}+$ MMT and PP + lcMMT.

Table 2. Fatigue in a Slow Load-Cycling Experiment (Cycle Time $4 \mathrm{~min}$ ) Estimated from Exponential Fits ${ }^{a}$

\begin{tabular}{lcccc}
\multicolumn{1}{c}{ sample } & $\tau_{\sigma}[\mathrm{min}]$ & $\tau_{L}[$ days $]$ & $\tau_{e}[\mathrm{~h}]$ & $\tau_{\Delta L}[\mathrm{~min}]$ \\
$\mathrm{PP}$ & 39 & 23.1 & 5.8 & 110 \\
$\mathrm{PP}+\mathrm{MMT}$ & 30 & 12.4 & 0.9 & 43 \\
$\mathrm{PP}+$ lcMMT & 34 & 8.4 & 1.7 & 50 \\
$\mathrm{PP}+\mathrm{hcMMT}$ & 43 & 6.1 & {$[1.0]$} & {$[140]$}
\end{tabular}

${ }^{a_{T}}$ The lifetimes $\tau_{\sigma}, \tau_{L}, \tau_{e}$, and $\tau_{\Delta L}$ characterize the macroscopic stress fatigue, the nanoscopic long-period fatigue, the nanoscopic lamellaextension fatigue, and the nanoscopic structure-heterogeneity fatigue, respectively. Data in brackets are only based on a part of the curve (cf. Figure 18).

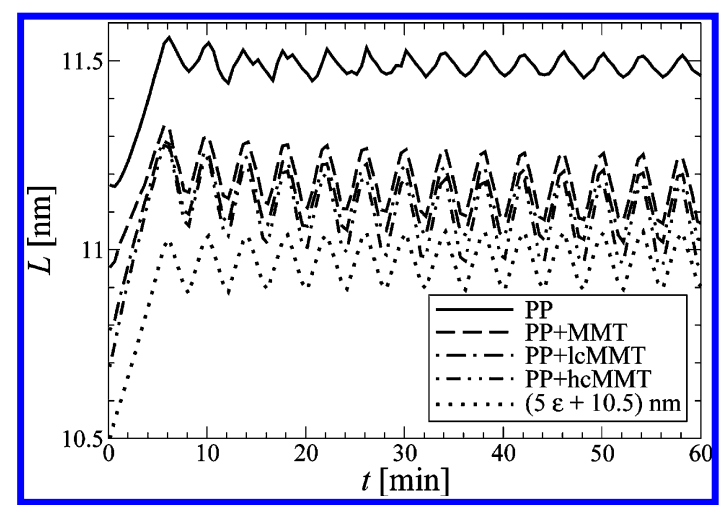

Figure 14. Nanostructure evolution during load cycling of PP nanocomposites determined from the CDF long-period peak. Mostfrequently long period $L(t)$ as a function of the elapsed time $t$. Additionally, the local macroscopic strain $\varepsilon(t)$ is shown.

nanostructure fatigue (Table 2). Because the data are already linear in a semilogarithmic plot, a residual does not need to be subtracted here.

Table 2 shows that the lifetime $\tau_{L}$ of the long-period decay is halved as MMT is blended in. Addition of the full amount of compatibilizer weakens the semicrystalline nanostructure of the polypropylene even more. The decay is accelerated by another factor of 2. Thus, the introduction of MMT and compatibilizer reduce the polypropylene self-reinforcement to $25 \%$ of its initial performance. The effective performance of the materials can be estimated from the lifetime of the macroscopic stress decay, $\tau_{\sigma}$. Table 2 shows that the blend has a reduced performance of 


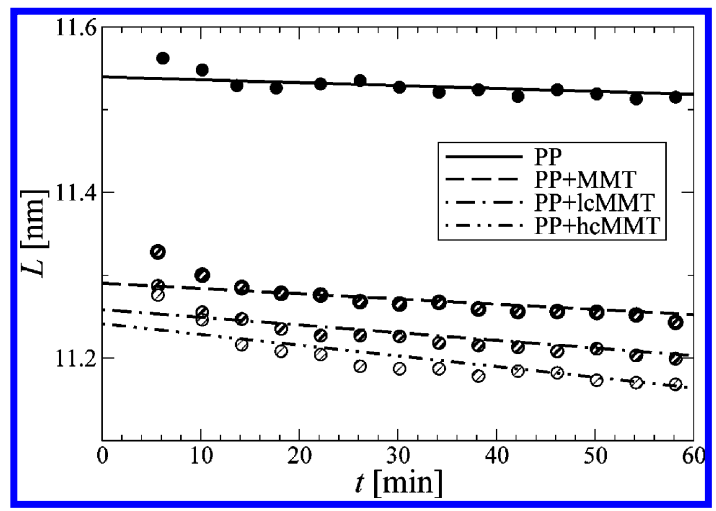

Figure 15. Assessment of nanostructure fatigue. Fit of the oscillation maxima from Figure 13 by an exponential for determination of the lifetime $\tau_{L}$ of long period decay.

$100 \times 21 / 27 \cong 77 \%$ with respect to the commercial polypropylene grade. Only the compatibilization of the MMT with $8 \%$ amphiphilic block copolymer leads to a slight performance gain to $111 \%$.

The dynamic variation of the lateral extension $e_{12}(t)$ of the layers during load cycling is presented in Figure 16. The initial

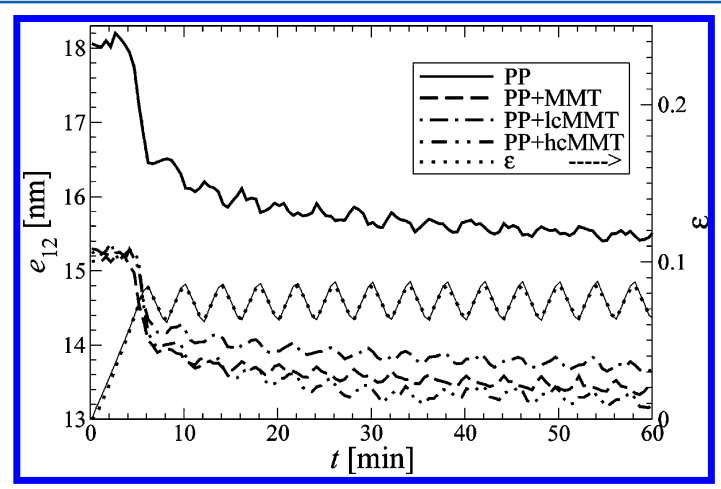

Figure 16. Nanostructure evolution during tensile testing of PP nanocomposites determined from the CDF long-period peak. Average lateral extension $e_{12}(t)$ of the crystalline PP domains as a function of the elapsed time $t$. Additionally the macroscopic strain $\varepsilon(t)$ is shown.

levels are somewhat higher than those reported from the plain tensile experiments, but this is only related to different data preevaluation. For this analysis we had to take out a step, namely an extrapolation of the scattering intensity to high angles. The extrapolation proved not to be stiff enough, and the noise introduced by extrapolation was higher than the weak variation of $e_{12}(t)$.

Layer-extension reduction starts after 4 min at $\varepsilon \approx 0.06$ and $\sigma \approx 27 \mathrm{MPa}$ for all studied materials. Thus, the lag time of lamellae disruption is not changed in the MMT-containing materials with respect to pure PP.

When the cycling starts, all materials respond with small oscillations about a monotonous decay. Thus, the fatigue of the nanostructure with respect to the breaking of crystalline layers can, again, be analyzed by exponential regression. Because the oscillations are not well-expressed but we know that we have eight SAXS patterns per cycle, this time the data are prepared for regression by sampling eight points in a running average. A residual layer extension, $e_{r}=13.2 \mathrm{~nm}$, is subtracted in order to linearize the data sets in a semilogarithmic plot. An exception is the curve of PP + hcMMT. It cannot be linearized over the full length of $85 \mathrm{~min}$, and the data look strange for $t<40 \mathrm{~min}$. The corresponding lifetimes $\tau_{e}$ of the lamellae extension are reported in Table 2 . The blending with MMT reduces the lifetime of the lamellae extension to $15 \%$, but in the composite $\mathrm{PP}+\mathrm{lcMMT}$ the lifetime has recovered to $30 \%$ of the initial value. The determined value for PP + hcMMT is based on a short interval $(t>40 \mathrm{~min})$. Thus, its significance is questionable, and in the table the value is enclosed in brackets.

Figure 16 exhibits a peculiar variation of the phase of the responses $e_{12}(t)$ on the dynamic strain $\varepsilon(t)$. For samples that are no composites (PP, PP + MMT) the layer extensions are low at the peak strains and vice versa. On the other hand, for the composites (PP + lcMMT, PP + hcMMT) the macroscopic stimulus $\varepsilon(t)$ and the nanoscopic response $e_{12}(t)$ are in phase. An explanation may be given that is based on a result of an earlier load cycling study of pure $\mathrm{PP}^{53}$ combined with the notion that in a composite matrix and filler are well bonded. In the earlier study the transition from strain-induced crystallization to crystallite disruption was found at $\sigma \approx 20 \mathrm{MPa}$. In the present load-cycling study, macroscopically the peak stress is well above the transition threshold. Thus, in pure PP and the blend many crystallites break. During the elongational branch of the cycles pieces of lamellae are moved apart. They recombine in the relaxational branch of the cycle. In the wellbonded composite the MMT bears part of the load, thus saving the polypropylene lamellae from breaking. On the other hand, the fraction of the load that is exerted on the polypropylene is low enough to guarantee a dominance of strain-induced crystallization over lamella disruption.

Figure 17 shows the breadth parameter $\Delta L=3 \sigma_{3}\left(h_{c a}\right)$ of the long-period distribution $h_{c a}$ as a function of time. It is related to

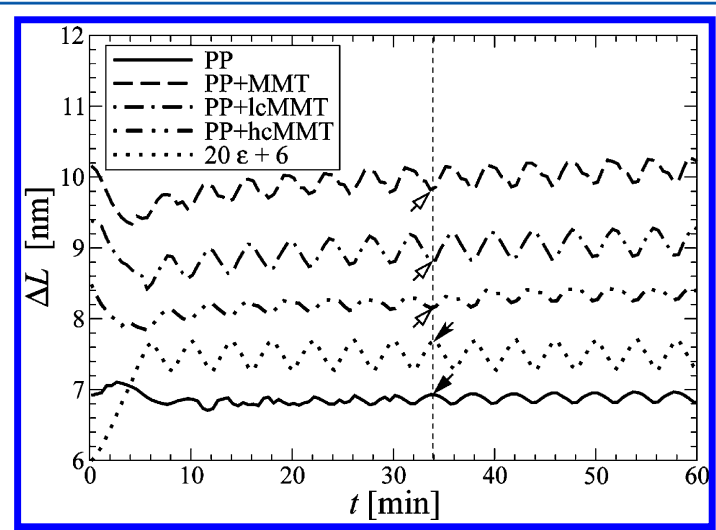

Figure 17. Nanostructure evolution during load cycling of PP nanocomposites determined from the CDF long-period peak. Breadth $\Delta L(t)$ of the long period distribution. Additionally, the local macroscopic strain $\varepsilon(t)$ is shown.

the variability of the distances in straining direction between two crystallites. During the first straining the MMT materials exhibit decreasing heterogeneity of the nanostructure in straining direction, whereas it almost remains constant for sample PP. This has already been found in tensile testing (cf. Figure 10).

As a function of time sample PP exhibits during the first seven cycles small variations about a constant level $\Delta L \approx 6.8 \mathrm{~nm}$. Thereafter, the material starts to respond to the macroscopic strain $\varepsilon(t)$ by a clear low-amplitude oscillation of $\Delta L(t)$. It is in phase with $\varepsilon(t)$. Thus, the arrangement of crystallites becomes more heterogeneous in each straining branch and returns to a more homogeneous state during relaxation. There is little fatigue 
related to the arrangement of crystallites. This behavior is readily explained by nonaffine straining of layer stacks that contain durable crystallites in which the low long-period stacks are less extensible than the high ones.

Compared to sample PP, the behavior of the MMT materials is characterized by four features. First, they show higher heterogeneity. Second, they respond from the beginning by a high-amplitude oscillation. Third, the phase of the oscillation is inverted with respect to the stimulus $\varepsilon(t)$ (cf. arrows in Figure 17). Thus, the distances between the crystallites become more uniform in each straining branch and relax into a more inhomogeneous state. A possible explanation is based on the assumption of a considerable fraction of undersized, premature crystallites that are too weak to withstand even moderate strain. Disintegration by chain unfolding leads to a loss of long periods at small $r_{3}$ in $h_{c a}\left(r_{12}, r_{3}\right)$, and a carryover to greater $r_{3} . h_{c a}$ becomes more uniform. During the relaxation branch the corresponding chains fold again to form an undersized crystal, and the breadth of $h_{c a}$ broadens again. Fourth, the heterogeneity of the MMT samples is increasing from cycle to cycle, exhibiting nanostructure fatigue. The reason for this nanostructure fatigue remains speculative. Let us assume that the premature state of the semicrystalline morphology itself is the reason. Then, under dynamic load it is easily worn down, leaving behind a broad spectrum of different long periods.

The corresponding lifetime analysis based on running averages $\overline{\Delta L}(t)$ of $\Delta L(t)$ is presented in Figure 18. It shows the

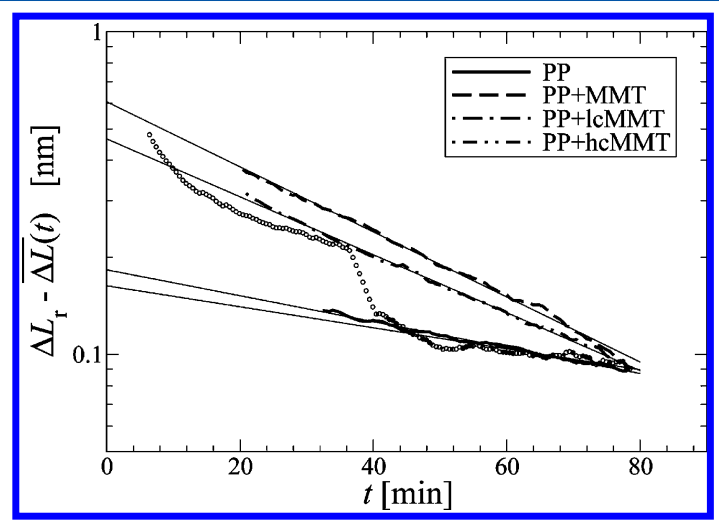

Figure 18. Nanostructure heterogeneity fatigue. Determination of lifetimes $\tau_{\Delta L} . \overline{\Delta L}(t)$ is the running average of the curves from Figure 17. Small circles show the complete set of PP + hcMMT containing the strange breakdown.

strange breakdown in the data from sample PP + hcMMT (full data set marked by circles), the running-average curves, and their fit by an exponential. The corresponding lifetimes $\tau_{\Delta L}$ are documented in Table 2. Obviously, the increase of nanostructure heterogeneity under dynamic load is doubled by addition of MMT to PP. The compatibilizer in the composites helps to reduce this fatigue.

The evolution of the scattering power $Q$ under dynamical load is almost trivial. The curves do not change significantly during the cycling. This means at least that there is not much change of contrast or void content during the load cycling.

Finally, an oversimplified cartoon of the nanoscale morphology is presented in Figure 19. It shows a rather well-developed lamellar structure in the polypropylene and in the materials that contain MMT a distorted layer structure that is reinforced by the silicate layers depicted as thin vertical domains.

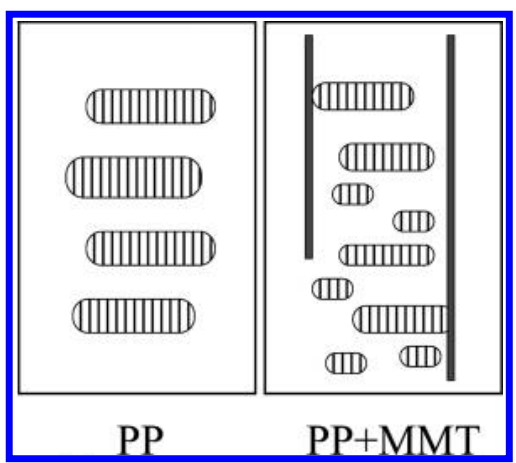

Figure 19. Simplified structural model for the semicrystalline structure of the pure polypropylene (left) and of the polypropylene phase in the nanocomposites (right). The thin vertical domains are drawn only to indicate that the composite contains MMT layers.

\section{CONCLUSIONS}

In general, the influences of the additives in a composite on the expression of the semicrystalline morphology of the matrix polymer are rarely taken into account. In-situ monitoring by means of small-angle X-ray scattering has shown that additives may vary the nanostructure at least of polypropylene considerably. On the one hand, this means that an improvement of mechanical properties in a composite may be hard to predict without having studied the interactions of the components. On the other hand, this shows that there is a considerable potential for tailoring the mechanical properties in the multidimensional parameter space. SAXS monitoring may be used as a tool to investigate this parameter space.

Here SAXS has been used to monitor slow mechanical tests of a set of nanocomposites from polypropylene (PP) and a layered silicate (montmorillonite, MMT). By comparing the extracted nanostructure evolution information to the mechanical data, it has been found that missing improvement of mechanical properties appears to result predominantly from the inhibition of a load-bearing semicrystalline morphology inside the PP by the MMT. Chemical compatibilization ${ }^{72-74}$ appears to be a secondary effect with the studied polypropylene grade. In fact, the predominant deterioration of mechanical properties of PP by nucleating mineral fillers has been reported in the literature. ${ }^{75}$ Load cycling the materials below the yield exhibits macroscopic and nanoscopic fatigue detected from a decay of the peak stresses and peak nanostructure parameters, respectively. From the respective lifetimes we have for the first time assessed the reinforcement of the composite and the weakening of the PP by the MMT. It has been found that crystallite growth is obstructed even more when MMT is compatibilized. It remains to be clarified if this effect is caused by the compatibilizer itself or by improved exfoliation of the MMT.

With respect to application the results indicate that the optimization of a commercial PP grade undertaken by the manufacturer may be lost when it is bonded to a filler with nucleating capacity. If the optimization of the formulation has to be redone, one could start from a PP grade without nucleators.

It should be mentioned that crystallite growth in PP may not only be limited by a high nucleating capacity. Also, the confinement of PP by nanoclay layers ${ }^{76}$ may lead to undersized crystallites. If there should be no way to control the nuclei density in MMT-extended polypropylene, then special processing, e.g., oscillating shear flow injection molding, ${ }^{77}$ could be considered to enhance the properties of the nanocomposite, but this may become too expensive for general application. 


\section{ACKNOWLEDGMENTS}

The authors thank the Hamburg Synchrotron Radiation Laboratory (HASYLAB) for beam time granted in the frame of projects II-2008-0015 and I-2011-0087. Kuraray Co., Ltd., Elastomer Division, Japan, is acknowledged for providing Kraton L-1203. This work has been supported by the seventh framework program of the European Union (Project NANOTOUGH FP7-NMP-2007-LARGE).

\section{REFERENCES}

(1) Varga, J. In Karger-Kocsis, J., Ed.; Polypropylene: Structure, Blends and Composites: Chapman \& Hall: London, 1995; pp 51-106.

(2) Edwards, K. L. Mater. Des. 2004, 25, 529-533.

(3) Youssef, Y.; Denault, J. Polvm. Compos. 1998, 19, 301-309.

(4) Gupta, V. B.; Mittal, R. K.; Sharma, P. K.; Mennig, G.; Wolters, I. Polvm. Compos. 1989, 10, 16-27.

(5) Pozsgay, A.; Fráter, T.; Papp, L.; Sajó, I.; Pukánszky, B. J. Macromol. Sci., Part B: Phys. 2002, 41, 1249-1265.

(6) Vu, Y. T.; Rajan, G. S.; Mark, J. E. Polvm. Int. 2004, 53, 10711077.

(7) Ma, J.; Zhang, S.; Qi, Z.; Li, G.; Hu, Y. I. Appl. Polvm. Sci. 2001, 83, 1978-1985.

(8) Li, J.; Zhou, C.; Gang.; Wang. Polvm. Test. 2003, 22, 217-223.

(9) Xu, W.; Liang.; Guodong.; Zhai, H.; Tang, S.; Hang, G.; Pan, W.-P. Eur. Polvm. I. 2003, 39, 1467-1474.

(10) Deshmane, C.; Yuan, Q.; Perkins, R. S.; Misra, R. D. K. Mater. Sci. Eng. A 2007, 458, 150-157.

(11) Smit, I.; Musil, V.; Švab, I. I. Appl. Polvm. Sci. 2004, 91, 40724081

(12) Purohit, P. J.; Huacuja-Sánchez, J. E.; Wang, D.-Y.; Emmerling, F.; Thünemann, A.; Heinrich, G.; Schönhals, A. Macromolecules 2011, 44, 4342-4354.

(13) Hussain, F.; Hojjati, M.; Okamoto, M.; Gorga, R. E. L. Compos. Mater. 2006, 40, 1511-1575.

(14) Jancar, J.; Douglas, J. F.; Starr, F. W.; Kumar, S. K.; Cassagnau, P.; Lesser, A. J.; Sternstein, S. S.; Buehler, M. I. Polvmer 2010, 51, 3321-3343.

(15) Folkes, M. J. In Karger-Kocsis, J., Ed.; Polypropylene: Structure, Blends and Composites: Chapman \& Hall: London, 1995; pp 340-370.

(16) Xu, J.; Srinivas, S.; Marand, H.; Agarwal, P. Macromolecules 1998, 31, 8230-8242.

(17) Hugel, T.; Strobl, G.; Thomann, R. Acta Polvm. 1999, 50, 214217.

(18) Strobl, G. Prog. Polvm. Sci. 2006, 31, 398-442.

(19) Compostella, M.; Coen, A.; Bertinotti, F. Angew. Chem. 1962, $74,618-624$.

(20) Norton, D. R.; Keller, A. Polvmer 1985, 26, 704-716.

(21) Olley, R. H.; Bassett, D. C. Polvmer 1989, 30, 399-409.

(22) Blomenhofer, M.; Ganzleben, S.; Hanft, D.; Schmidt, H.-W.; Kristiansen, M.; Smith, P.; Stoll, K.; Mäder, D.; Hoffmann, K. Macromolecules 2005, 38, 3688-3695.

(23) Kristiansen, P. M.; Gress, A.; Hanft, D.; Schmidt, H.-W. $\underline{\text { Polvmer }}$ 2006, 47, 249-253.

(24) Libster, D.; Aserin, A.; Garti, N. Polvm. Adv. Technol. 2007, 18, 685-695.

(25) Lipp, J.; Shuster, M.; Terry, A. E.; Cohen, Y. Polvm. Eng. Sci. 2008, 48, 705-710.

(26) Li, J.; Ton-That, M.-T.; Leelapornpisit, W.; Utracki, L. A. Polvm. Enc. Sci. 2007, 47, 1447-1458.

(27) Thorby, D. Structural Dynamics and Vibration in Practice, 1st ed.; Butterworth-Heinemann: Amsterdam, 2008.

(28) Peterlin, A. Annu. Rev. Mater. Sci. 1972, 2, 349-380.

(29) Takemori, M. T. Annu. Rev. Mater. Sci. 1984, 14, 171-204.

(30) Crist, B. Annu. Rev. Mater. Sci. 1995, 25, 295-323.

(31) Pegoretti, A. In Karger-Kocsis, J., Fakirov, S., Eds.; Nano- and Micro-Mechanics of Polymer Blends and Composites; Hanser: Munich, 2009; pp 301-339.
(32) Brown, H. R.; Kramer, E. J.; Bubeck, R. A. I.Mater. Sci. 1988, 23, $248-252$.

(33) Toki, S.; Sics, I.; Burger, C.; Fang, D.; Liu, L.; Hsiao, B. S.; Datta, S.; Tsou, A. H. Macromolecules 2006, 39, 3588-3597.

(34) Jankova, K.; Chen, X.; Kops, J.; Batsberg, W. Macromolecules 1998, 31, 538-541.

(35) Jankova, K.; Kops, J.; Chen, X.; Batsberg, W. Macromol. Rapid Commun. 1999, 20, 219-223.

(36) Stribeck, N.; Nöchel, U.; Funari, S. S.; Schubert, T. J. Polym. Sci. Polvm. Phvs. 2008, 46, 721-726.

(37) Stribeck, N. In Karger-Kocsis, J., Fakirov, S., Eds.; Nano- and Micromechanics of Polymer Blends and Composites; Hanser Publisher: Munich, 2009; Vol. 1, pp 269-300

(38) Denchev, Z.; Dencheva, N.; Funari, S. S.; Motovilin, M.; Schubert, T.; Stribeck, N. J. Polym. Sci., Part B: Polvm. Phys. 2010, 48, 237-250.

(39) Stribeck, N. X-Ray Scattering of Soft Matter; Springer: Heidelberg, 2007.

(40) Stribeck, N. I. Appl. Crustallogr. 2001, 34, 496-503.

(41) Ruland, W. Colloid Polym. Sci. 1977, 255, 417-427.

(42) Vonk, C. G. Colloid Polvm. Sci. 1979, 257, 1021-1032.

(43) Debye, P.; Bueche, A. M. I. Appl. Phvs. 1949, 20, 518-525.

(44) Porod, G. Colloid Polvm. Sci. 1951, 124, 83-114.

(45) Vonk, C. G. I. Appl. Crustallogr. 1973, 6, 81-86.

(46) Baltá Calleja, F. J.; Vonk, C. G. X-Ray Scattering of Synthetic Polymers; Elsevier: Amsterdam, 1989.

(47) Brown, H. R.; Kramer, E. J. J. Macromol. Sci., Part B: Phys. 1981, 19, 487-522.

(48) Karl, A.; Cunis, S.; Gehrke, R.; Krosigk, G. v.; Lode, U.; Luzinov, I.; Minko, S.; Pomper, T.; Senkovsky, V.; Voronov, A.; Wilke, W. J. Macromol. Sci., Part B: Phys. 1999, 38, 901-912.

(49) Pomper, T.; Lode, U.; Karl, A.; Krosigk, G. V.; Minko, S.; Luzinov, I.; Senkovsky, V.; Voronov, A.; Wilke, W. J. Macromol. Sci. Phvs. 1999, B38, 869-883.

(50) Kobayashi, H.; Shioya, M.; Tanaka, T.; Irisawa, T. Compos. Sci. Technol. 2007, 67, 3209-3218.

(51) Fischer, S.; Diesner, T.; Rieger, B.; Marti, O. I. Appl. Crustallogr. 2010, 43, 603-610.

(52) Statton, W. O. I. Polvm. Sci. 1962, 58, 205-220.

(53) Stribeck, N.; Nöchel, U.; Funari, S. S.; Schubert, T.; Timmann, A. Macromol. Chem. Phys. 2008, 209, 1992-2002.

(54) Peterlin, A. Text. Res. I. 1972, 42, 20-30.

(55) Stribeck, N.; Androsch, R.; Funari, S. S. Macromol. Chem. Phys. 2003, 204, 1202-1216.

(56) Fronk, W.; Wilke, W. Colloid Polvm. Sci. 1985, 263, 97-108.

(57) Stribeck, N. J. Macromol. Sci., Part C: Polvm. Rev. 2010, 50, 4058.

(58) Stribeck, N.; Zeinolebadi, A.; Ganjaee Sari, M.; Frick, A.; Mikoszeg, M.; Botta, S. Macromol. Chem. Phys. 2011, 212, 2234-2248.

(59) Peterlin, A. I. Mater. Sci. 1971, 6, 490-508.

(60) Porod, G. Fortschr. Hochpolvm.-Forsch. 1961, 2, 363-400.

(61) Wilkes, G. L.; Zhou, H. I. Mater. Sci. 1998, 33, 287-303.

(62) Butler, M. F.; Donald, A. M. Macromolecules 1998, 31, 62346249.

(63) Wu, J.; Schultz, J. M.; Yeh, F.; Hsiao, B. S.; Chu, B. Macromolecules 2000, 33, 1765-1777.

(64) Barbi, V.; Funari, S. S.; Gehrke, R.; Scharnagl, N.; Stribeck, N. Macromolecules 2003, 38, 749-758.

(65) Kawakami, D.; Ran, S.; Burger, C.; Avila Orta, C.; Sics, I.; Chu, B.; Hsiao, B. S.; Kikutani, T. Macromolecules 2006, 39, 2909-2920.

(66) Miyazaki, T.; Hoshiko, A.; Akasaka, M.; Shintani, T.; Sakurai, $\underline{S}$. Macromolecules 2006, 39, 2921-2929.

(67) Ibanes, C.; David, L.; de Boissieu, M.; Séguéla, R.; Epicier, T.; Robert, G. J. Polym. Sci. Part B: Polvm. Phys. 2004, 42, 3876-3892.

(68) Todorov, L. V.; Viana, J. C. L.Appl. Polvm. Sci. 2007, 106, 16591669.

(69) Drozdov, A. D.; Christiansen, J. d. Eur. Polvm. I. 2007, 43, $10-25$.

(70) Ahmed, S.; Jones, F. R. I. Mater. Sci. 1990, 25, 4933-4942. 
(71) Sumita, M.; Tsukumo, Y.; Miyasaka, K.; Ishikawa, K. I. Mater. Sci. 1983, 18, 1758-1764.

(72) Marchant, D.; Jayaraman, K. Ind. Eng. Chem. Res. 2002, 41, 6402-6408.

(73) Rohlmann, C. O.; Horst, M. F.; Quinzani, L. M.; Failla, M. D. Eur. Polvm. I. 2008, 44, 2749-2760.

(74) Santos, K. S.; Liberman, S. A.; Oviedo, M. A. S.; Mauler, R. $\underline{\text { S. }}$ Composites, Part A 2009, 40, 1199-1209.

(75) McGenity, P. M.; Hooper, J. J.; Paynter, C. D.; Riley, A. M.; Nutbeem, C.; Elton, N. J.; Adams, J. M. Polvmer 1992, 33, 5215-5224.

(76) Jin, Y.; Rogunova, M.; Hiltner, A.; Baer, E.; Nowacki, R.; Galeski, A.; Piorkowska, E. J. Polym. Sci. Part B: Polvm. Phvs. 2004, 42, 3380-3396.

(77) Chen, Y. H.; Zhong, G. J.; Wang, Y.; Li, Z. M.; Li, L. Macromolecules 2009, 42, 4343-4348. 